\title{
Evaluating models of gesture and speech production for people with aphasia
}

\author{
(to appear in Cognitive Science)
}

Carola de Beer ${ }^{1}$, Katharina Hogrefe ${ }^{2}$, Martina Hielscher-Fastabend ${ }^{3}$, \& Jan P. de Ruiter ${ }^{4}$

${ }^{1}$ Cognitive Sciences, Linguistics Department, University of Potsdam, Potsdam, Germany, ${ }^{2}$ Clinical Neuropsychology Research Group (EKN), Institute of Phonetics and Speech Processing, Ludwig-Maximilians-Universität München, Munich, Germany, ${ }^{3}$ Clinical Linguistics, Department of Linguistics and Literature Science, Bielefeld University, Bielefeld, Germany, ${ }^{4}$ Departments of Psychology and Computer Science, Tufts University, Medford, MA, USA

Keywords: aphasia, gesture production, model, iconic gestures, communication

Running head: Testing gesture production models for PWA

Corresponding author:

Dr. Carola de Beer

Cognitive Sciences, Linguistics Department, University of Potsdam, Campus Golm, KarlLiebknecht-Straße 24-25, 14476 Potsdam

carola.de.beer@uni-potsdam.de

\begin{abstract}
People with aphasia use gestures not only to communicate relevant content but also to compensate for their verbal limitations. The Sketch Model (De Ruiter, 2000) assumes a flexible relationship between gesture and speech with the possibility of a compensatory use of the two modalities. In the successor of the Sketch Model, the AR-Sketch Model (De Ruiter, 2017), the relationship between iconic gestures and speech is no longer assumed to be flexible and compensatory, but instead iconic gestures are assumed to express information that is redundant to speech. In this study, we evaluated the contradictory predictions of the Sketch Model and the AR-Sketch Model using data collected from people with aphasia as well as a group of people without language impairment. We only found compensatory use of gesture in the people with aphasia, whereas the people without language impairments made very little compensatory use of gestures. Hence, the people with aphasia gestured according to the prediction of the Sketch Model, whereas the people without language impairment did not. We conclude that aphasia fundamentally changes the relationship of gesture and speech.
\end{abstract}




\section{Introduction}

Communication is an interactive and multimodal activity (Kendon, 2004). Speech is normally the dominant modality in natural communication, whereas gestures are defined as a speech-accompanying communicative modality (McNeill, 1985; McNeill, 1992). Gestures are mostly performed with the arms and hands and their meaning is closely related to the accompanying speech. For more thorough descriptions of gestures, different accounts have been put forward to subdivide gestures into different categories or types. One of the most influential systems to describe the range of characteristics within the category of gestures is Kendon's continuum (as described by McNeill, 1992). Following this approach, it was suggested that four different subtypes of gestures differ from one another with regard to their relation to speech, their linguistic properties and their degree of conventualisation. The distinguished categories are described as follows:

Gesticulations have a non-conventionalised (idiosyncratic) form-meaning relation, and only appear together with accompanying speech.

Emblems are gestures with a conventionalised (language-specific) form-meaning relation. Emblems can be used either with or without accompanying speech.

Pantomimes are defined as non-conventualised imitations of motor actions with some linguistic properties, mostly produced in the absence of speech.

Sign Languages are fully conventualised language systems with all linguistic properties and are, by definition, used to replace speech.

McNeill (1992) focused on the gesticulations from Kendon's continuum and used a semiotic approach to distinguish four different categories of speech-accompanying gestures from one another:

1) Iconic gestures represent aspects of the content of the accompanying speech by their shape and manner of their execution. They are semantically and temporally tightly related to speech, and their referents are concrete entities. Their realisation is not conventionalized, but idiosyncratic.

2) Like iconic gestures, metaphoric gestures are gestures that represent imagery idiosyncratically (not conventionalized). The difference with iconic gestures is that they refer to abstract content.

3) Deictic gestures are pointing movements. Concrete deictic gestures point to a concrete direction or object, whereas abstract deictic gestures are used to establish a point in space and associate it with a discourse referent.

4) Beats are small, repetitive, and rhythmic movements that do not represent specific elements from the speech.

De Ruiter (2000) also uses McNeill's (1992) classification scheme, with one modification: he does not distinguish between metaphoric gestures and iconic gestures, on 
the grounds that it doesn't matter for an iconic gesture if it refers to an abstract or a concrete entity.

\subsection{The relationship between (iconic) gestures and speech}

There are a variety of theories addressing the relationship of iconic gestures and speech, and these theories also make assumptions about the primary function of iconic gestures. It has been suggested that iconic gestures' primary function is to aid the speaker, i.e., to facilitate speech production (Butterworth \& Hadar, 1989; Krauss, Chen, \& Gottesman, 2000; Krauss, Morrel-Samuels, \& Colasante, 1991). This lexical facilitation hypothesis also states that gestures do not seem to play an important role for the intelligibility of an utterance (Krauss, Dushay, Chen, \& Rauscher, 1995).

Evidence against the lexical facilitation hypothesis and in favor of a communicative function of iconic gestures suggests that iconic gestures do play an important role in the transmission of content (Beattie \& Coughlan, 1999; Beattie \& Shovelton, 2011; Holler, Turner, \& Varcianna, 2013). The production of iconic gestures was shown to be influenced by the presence of a visible listener and therefore assumed to have a primarily social and communicative function.

A third view is that iconic gestures are multi-functional, in the sense that they both have a communicative function and internally facilitate speech production (Alibali, Heath, \& Myers, 2001; Alibali, Kita, \& Young, 2000). However, according to this view iconic gestures are assumed to support the conceptualisation and planning of a spoken utterance and not to facilitate word retrieval (Alibali et al., 2000; De Ruiter, 2006; Goldin-Meadow, 1999).

To shed more light on the relationship between gesture and speech, scholars have proposed theories and models of the processes involved in the production of gesture and speech (De Ruiter, 2000; Kita \& Özyürek, 2003; Krauss et al., 2000; McNeill \& Duncan, 2000).

Of the available models, the Sketch Model (de Ruiter, 2000) is the only one accommodating the production of the four major gesture types, namely iconic gestures, deictic gestures, emblems and pantomimes. De Ruiter extended Levelt's (1989) model of speech production to include the production of co-speech gesture (Fig. 1). Its four core assumptions on the relation between gesture and speech are: 1) The same communicative intention underlies the planning of both gesture and speech, 2) Gesture and speech are planned together in the conceptualizer, but executed separately by the two underlying processing modules, which are temporally coordinated by a signalling mechanism, 3) Gesture and speech are planned and produced to aid communication, i.e., to facilitate the interlocutor's understanding, and 4) Gesture and speech are assumed to be mutually adaptive, in the sense that one channel can compensate for expressive limitations in the other.

The planning of a multimodal utterance starts with a speaker's communicative intention. Based on this communicative intention, the conceptualizer retrieves the relevant information, i.e. representations from working memory. These representations are either propositional or imagistic in nature and are processed independently of one another by the conceptualizer. 


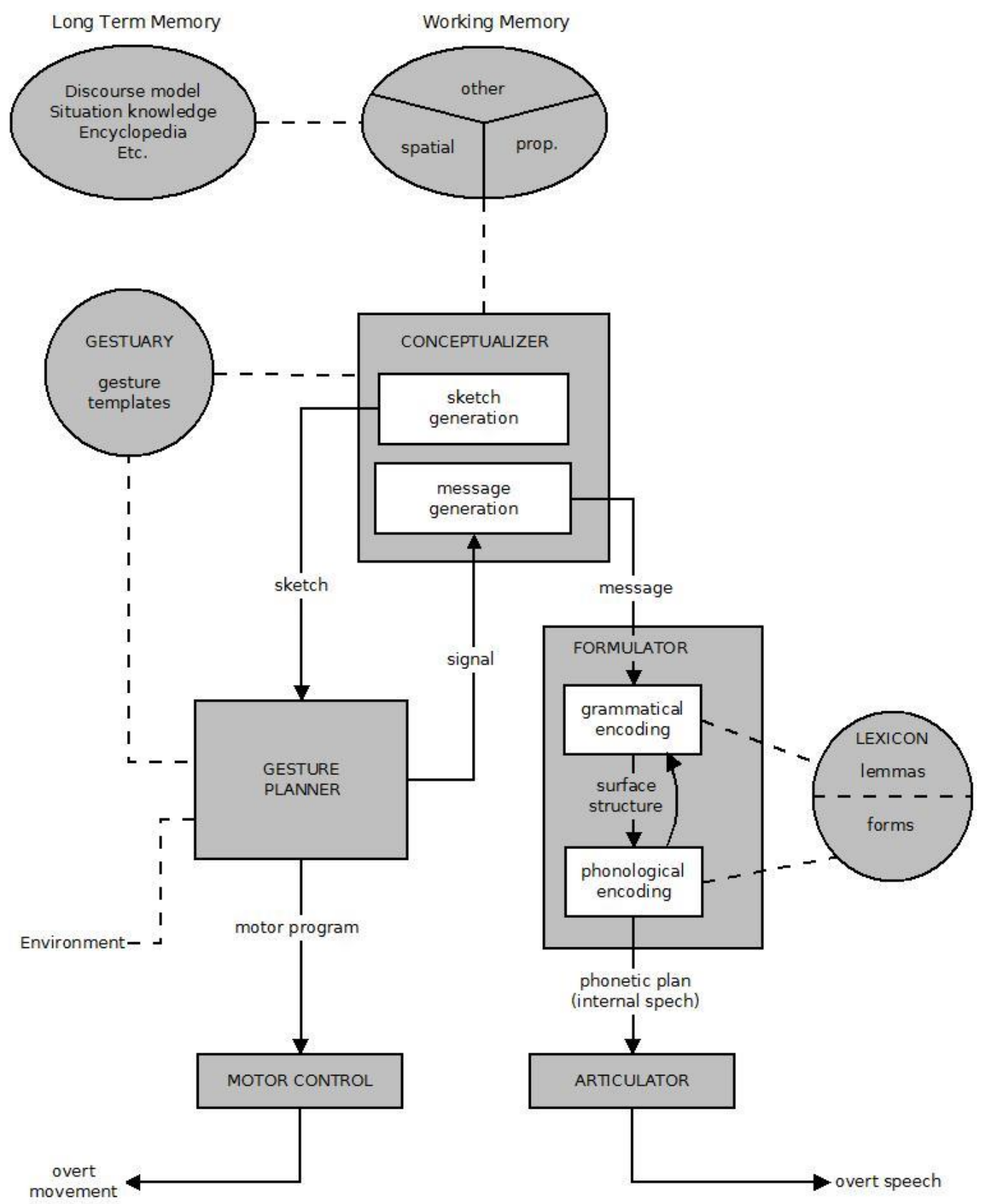

Figure 1. The Sketch Model for gesture and speech production (De Ruiter, 2000, p. 298). Reprinted from "The Production of Gesture and Speech" (p. 298), by J. de Ruiter, 2000, in Language and Gesture (pp. 284-311), D. McNeill (Ed.). Cambridge, United Kingdom: Cambridge University Press. Copyright (C) 2000 Cambridge University Press. Reprinted with permission.

The propositional representations are encoded into a preverbal message to be used to plan and produce the spoken part of the utterance (in line with Levelt's model of speech production). The imagery representations build the basis for the encoding of a Sketch, which is the equivalent of the preverbal message in the gesture modality. The sketch of an iconic gesture solely contains spatio-temporal and imagery representations retrieved from working memory. This Sketch is further processed and transformed in the gesture planner defining the more detailed aspects of its execution (e.g. which body parts are used for the gesture). The Gesture Planner encodes the transformed information into a motor program. 
This motor program is sent to the motor control to be converted into the overt movement, i.e. the gesture. Since we focus on the production of iconic gestures in this paper, we will not describe the production processes of the other gesture types (please see De Ruiter (2000) for further details).

One of the core assumptions of the Sketch Model is the flexible relationship of gesture and speech, which allows for a compensatory use of the two modalities. This assumption has been referred to as the tradeoff hypothesis (De Ruiter, Bangerter, \& Dings, 2012). In contrast to the tradeoff hypothesis, the hand-in-hand hypothesis assumes that gesture and speech are used to express the same aspects of content (So, Kita, \& GoldinMeadow, 2009). To investigate these contradicting hypotheses, De Ruiter, Bangerter, and Dings (2012) examined the production of deictic and iconic gestures in a director-matcher task with undergraduate students from Radboud University in the Netherlands, manipulating a) the mutual visibility between speaker and hearer, b) the codability of the stimuli (i.e., the difficulty of verbally describing the referent) and c) common ground. For the iconic gestures, they found no evidence in favour of the tradeoff hypothesis. The use of iconic gestures neither increased with decreasing codability of the target stimuli, nor decreased with repetition of the stimuli, i.e. with increasing common ground. Visibility was shown to influence the use of so-called "obligatory" iconic gestures, defined as gestures that the listener needs to see in order to understand the verbal utterance they accompany. Not surprisingly, the use of obligatory iconics decreased in the case of blocked visibility between speaker and listener. In summary, De Ruiter et al.'s results strongly supported the assumptions of the hand-in-hand hypothesis on the relationship of iconic gestures and the accompanying speech.

To accommodate the results described above, De Ruiter (2017) revised the Sketch Model's core assumptions, resulting in the Asymmetric Redundancy (AR) Sketch Model. This model is the same as the Sketch model, except for the Conceptualizer. In the AR-Sketch Model the two communicative modalities are not assumed to be mutually adaptive, as is the case in the original Sketch model. Instead, speech is assumed to be the primary modality for communicating content. The gesture channel is assumed to express content that is redundant with the communicative message in the speech channel, unless this information is an unavoidable side effect of the execution of the gesture (see De Ruiter 2017 for details). To incorporate these assumptions in the framework of the Sketch Model, the information selection process in the conceptualizer was changed (see Fig. 2). In the original Sketch Model, the imagery and the propositional information are retrieved from working memory and independently represented in the conceptualizer. The generation of the preverbal message and the Sketch are therefore based on independent sources of information. In the AR-Sketch Model, however, the imagery information retrieved from working memory for the gesture is a subset of the propositional information that is used for generating speech. So, while the speech is planned based on the complete set of available information, the gesture is based on a subset of this information. This incorporates and explains the common finding that understanding speech without having access to gesture is much easier than understanding gesture without having access to speech. 

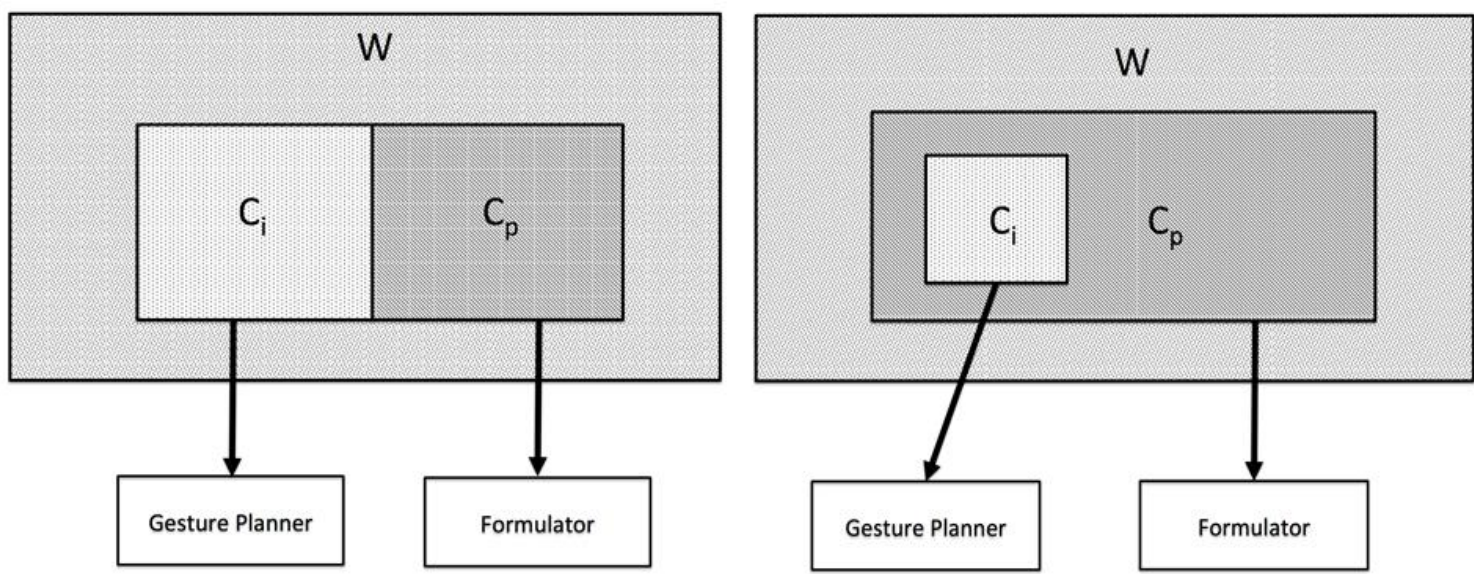

Figure 2. The structure of the conceptualiser in the original Sketch Model (on the left) and in the AR-Sketch Model (on the right). $W=$ working memory. $C$ = subset of representations selected from working memory to be part of the communicative intention. $C_{p}=$ propositional representations. $C_{i}=$ imagistic representations. Reprinted from: De Ruiter, J.P. (2017). The asymmetric redundancy of gesture and speech. In R. B. Breckinridge Church, M.W. Alibali, \& S.D. Kelly (Eds.), Why Gesture? How the hands function in speaking, thinking and communicating (S. 59-75), Amsterdam/Philadelphia: John Benjamins Publishing Company (https://benjamins.com/catalog/gs.7). Reprinted with permission.

De Ruiter (2017) still assumes gesture to have a primarily communicative function.

Even in cases of complete redundancy of gesture and speech, gesture serves a communicative function by reinforcing the intelligibility of the expressed content. However, one of the core assumptions of the original Sketch Model was revised in the AR-Sketch Model: Iconic gestures are not assumed to compensate for limitations in the verbal modality anymore.

\subsection{The relationship of (iconic) gestures and speech in people with aphasia}

People with aphasia face significant limitations in their production and reception of spoken and written language. Communicative modalities other than speech can therefore gain relevance in the case of aphasia (Anglade, Le Dorze, \& Croteau, 2020; Damico, Wilson, Simmons-Mackie, \& Tetnowski, 2008; Sekine \& Rose, 2013; Sekine, Rose, Foster, Attard, \& Lanyon, 2013). A key topic in studying gesture use in people with aphasia is the relationship between the speech and the gesture modality. In earlier studies, it was suggested that aphasia leads to a parallel breakdown of gesture and speech as well as other communicative modalities (Cicone, Wapner, Foldi, Zurif, \& Gardner, 1979; Duffy \& Duffy, 1981; Duffy \& Liles, 1979). The core assumption of this Asymbolia Hypothesis was that a central underlying deficit, named asymbolia, resulted in processing difficulties of symbols in different modalities (Duffy \& Liles, 1979). It was further assumed that a shared underlying deficit resulting in parallel disturbances of gesture and speech prevents people with aphasia from using gestures to effectively compensate for their verbal limitations (Cicone et al., 1979). When comparing the production of different gesture types by PWA in four tasks with varying linguistic constraints, Lott (1999) found the highest gesture frequency in conversation, which is the task with the lowest communicative constraints. PWA's gesture 
production decreased in the tasks with higher communicative constraints, namely a story retelling, picture description and a story completion task. Lott concluded that PWA use gestures most effectively with low linguistic constraints.

However, using gestures to express meaning by complementing or even replacing spoken production is of high relevance for people with aphasia, especially for people with severe expressive limitations. Therefore, many scholars have investigated the potential of co-speech gestures to be used for effective compensation by people with aphasia (Feyereisen, 1983; Herrmann, Reichele, Lucius-Hoene, Wallesch, \& Johannsen-Horbach, 1988). More recent studies have looked at the role of gestures for the expression of content by people with aphasia (De Beer et al., 2017; Hogrefe, Ziegler, Weidinger, \& Goldenberg, 2017; Hogrefe, Ziegler, Wiesmayer, Weidinger, \& Goldenberg, 2013; Wilkinson, Beeke, \& Maxim, 2010). A rating study conducted by Hogrefe et al. (2013) revealed that some people with aphasia, and especially people with severe types of aphasia, conveyed more information using gestures than they did using spoken utterances in a narration task. The authors concluded that people with aphasia compensate for their verbal limitations by employing gestures. Also using a rating paradigm, Rose, Mok \& Sekine (2017), focusing exclusively on pantomime gestures, confirmed the finding that people with aphasia use gestures to express information that complements verbal speech. They compared the information expressed by both gesture and speech in isolation and by both channels in combination. The combined and therefore most natural signal, consisting of both gesture and speech, was found to be most informative to the listeners. Following up on the study by Rose et al. (2017), de Beer and colleagues (2017) investigated the communicative role of three different gesture types, namely pantomimes, emblems and referential gestures (a category containing both iconic and deictic gestures) in extracts from spontaneous conversations. The results revealed that the naive raters understood the messages expressed by the PWA of varying severities more accurately when they were expressed using gesture and speech as opposed to speech alone. This was true for all gesture types under investigation, including iconic gestures. The spontaneous use of iconic gestures by people with aphasias of varying severities has also been described by other scholars (Kong, Law, Wat, \& Lai, 2015; Sekine et al., 2013) and people with aphasia were found to use iconic gestures to express different aspects of semantic content (De Beer, Hogrefe, \& De Ruiter, 2018; Dipper, Pritchard, Morgan, \& Cocks, 2015).

Taken together, the results of the aforementioned studies suggest that people with aphasia use gestures to add relevant content to their communication and thereby compensate for their limitations in speech. They employ various gesture types to express speech-complementing information, including iconic gestures. The assumption of a parallel impairment of gesture and speech in people with aphasia is not supported by these findings. In fact, these results support the assumption that gestures are employed by PWA to compensate for limitations in the verbal channel.

To summarize, it is debated whether PWA are able to employ gestures for effective compensation of their verbal difficulties. Furthermore, some authors found that PWA make less use of gestures in tasks with higher linguistic constraints. On the other hand, there is evidence suggesting that PWA use gestures, including iconic gestures, to express important content, and this was found to be particularly evident with increasing verbal limitations, i.e. more severe types of aphasia.

De Ruiter and De Beer (2013) discuss how well different models of gesture and speech production account for the finding that people with non-fluent aphasia show lower 
gesture rates per minute, but higher gesture-to-word ratios of iconic gestures compared to people without language impairments. They argued that the original Sketch Model (De Ruiter, 2000) can account for this finding and for the relationship between gesture and speech in the case of aphasia (please see De Ruiter \& De Beer (2013) for a thorough discussion of the Growth Point Theory (McNeill, 1985; McNeill, 1992), the Interface Model (Kita \& Özyürek, 2003) and the Lexical Access Model suggested by Krauss, Chen and Gottesman (2000)). In the framework of the Sketch Model, the conceptualizer of the PWA recognizes the deficit in speech production. It reacts to this productive deficit by adaptation and transfers more of the information to be expressed to the gesture modality. Therefore, parts of the information that people without language impairment express using speech are expressed using gesture by people with non-fluent aphasia. This is how De Ruiter and De Beer explained the planning processes underlying the compensatory gesture use of people with non-fluent aphasias.

The assumptions and predictions of the Sketch Model have so far only been discussed for people with non-fluent aphasia, whereas the AR-Sketch Model (De Ruiter, 2017) has not been tested with respect to PWA's production of gesture and speech at all.

In this study we aim to investigate how well the Sketch Model and the AR-Sketch Model account for the use of iconic gestures (iconics) produced by a group of people with aphasias of varying types and severities as opposed to a control group of people without language impairments.

The most ecologically valid task to use in this study is a free and unconstrained conversation with an interlocutor, as conversation is one of the central uses of language in social interaction. Spontaneous conversation allows for a flexible choice of topics and content to be expressed by the participants. However, our current research question clearly requires the inclusion of a second task that restricts the flexible choice of the content to be expressed and is thereby more challenging for the PWA with respect to the verbal expression of content. For this reason, we decided to use an additional task, namely the cartoon-narration task (McNeill, 1992). In this task the participant watches short fragments of a cartoon movie and subsequently tells another person about what happens in the cartoon fragment. So, the content to be described is given, which means that the participant has far less control over the nature of the utterances they are going to produce. Differences in the compensatory use of gesture are therefore expected to show up more clearly than in the free conversation task.

Since we aim to test the production of iconic gestures in relation to spoken production, we will analyse our results in terms of gesture-to-word ratios as opposed to gestures-per-minute, since we focus on the relationship of gesture and speech in the current study. This relationship is more appropriately reflected by the gesture-to-wordratios than by gesture rates per time unit. We define iconics following McNeill's (1992) definition of iconic gestures (see above), i.e., subsuming the categories of iconic gestures and pantomimes (note that some scholars, e.g. De Ruiter (2000) and Sekine \& Rose (2013) have classified pantomimes as a distinct category from iconic gestures, see De Beer, De Ruiter, Hielscher-Fastabend, \& Hogrefe $(2019)^{1}$ for separate analyses of the production of

\footnotetext{
${ }^{1}$ In De Beer et al. (2019), the authors studied the influence of varying communicative constraints on the relative distribution of PWA's production of different gesture types. That study and the current study are based on the same raw data, collected from PWA and neurotypical controls. The current study addresses a different research question than the
} 
iconic gestures and pantomimes). Note that we follow De Ruiter (2000) in not distinguishing metaphoric gestures as a distinct category from iconics (please see De Ruiter, 2000, p. 285 for a more detailed explanation).

The Sketch Model (De Ruiter, 2000) predicts that PWA will show higher iconic gesture-to-word-ratios than people without language impairments in both communicative tasks, and that gesture-to-word-ratios are higher with communicative constraints (in the narration task), because in that task, the need to compensate with gesture is higher. In contrast, the AR-Sketch Model (De Ruiter, 2017) predicts no effect of varying communicative constraints on the gesture-to-word-ratios of iconic gestures in either group of participants.

To systematically test these predictions on the potential compensatory use of iconic gestures for PWA, we studied gesture production in the two different communicative tasks with varying communicative constraints described above, in both PWA and controls.

\section{Method}

\subsection{Participants}

We conducted our experiment with 26 participants with aphasia (PWA) ( 9 females, 17 males) with a mean age of 56.31 years $(S D=10.44$; range $=36-85$ years $)$ as well as a group of 26 control participants (CP) without any neurological, psychiatric or communicative impairments. Each of the CP was matched one-to-one to one of the 26 PWA with respect to gender and age (with a maximum difference of five years of age). The CP's mean age was 56.38 ( $S D=10.58$; range $=34-85$ years $)$. Nine of the CP were female and 17 were male.

For the group of PWA, we included participants with aphasia severities ranging from mild to severe in the post-acute or chronic stage and with German as their native language (PWA17 was an exception to this, since she acquired German as her early L2). Exclusion criteria were the presence of an additional neurological (e.g. multiple sclerosis) or psychiatric disease (e.g. depression), since these could influence the participant's communicative and interactive behaviour. Furthermore, participants with impairments in their visual processing were excluded from the study. The PWA had varying etiologies of their aphasias and the time post onset ranged from 6 to 318 months (see Table 1).

2019 study, namely the communicative load distribution between speech and (specifically) iconic gestures, for a comparative evaluation of the Sketch model and the AR-Sketch model. This research question required the analysis of the rate of iconic gestures per 100 words. 
Table 1. Participant information of the participants with aphasia (PWA) including demographic data and the aphasia types according to the AAT.

\begin{tabular}{cccccc}
\hline $\begin{array}{c}\text { Participant } \\
\text { ID }\end{array}$ & Sex & Age & Etiology & $\begin{array}{c}\text { Time post onset (n } \\
\text { of months) }\end{array}$ & Aphasia Type \\
\hline PWA01 & $\mathrm{f}$ & 57 & $\mathrm{Cl}(\mathrm{L})$ & 235 & Broca \\
PWA02 & $\mathrm{m}$ & 70 & hypoxia & 88 & Wernicke \\
PWA04 & $\mathrm{f}$ & 67 & $\mathrm{TBI}$ & 10 & Broca \\
PWA07 & $\mathrm{m}$ & 54 & $\mathrm{Cl}(\mathrm{L})$ & 6 & Global \\
PWA08 & $\mathrm{m}$ & 58 & $\mathrm{Cl}(\mathrm{L})$ & 177 & Broca \\
PWA09 & $\mathrm{m}$ & 59 & $\mathrm{Cl}(\mathrm{L})$ & 96 & Wernicke \\
PWA10 & $\mathrm{m}$ & 44 & $\mathrm{Cl}(\mathrm{L})$ & 212 & Anomic \\
PWA11 & $\mathrm{m}$ & 53 & $\mathrm{Cl}(\mathrm{L})$ & 22 & Anomic \\
PWA12 & $\mathrm{m}$ & 61 & $\mathrm{Cl}(\mathrm{R})$ & 13 & Global \\
PWA13 & $\mathrm{m}$ & 85 & $\mathrm{Cl}(\mathrm{THA})$ & 19 & Wernicke \\
PWA15 & $\mathrm{m}$ & 48 & $\mathrm{Cl}(\mathrm{L})$ & 177 & Wernicke \\
PWA17 & $\mathrm{f}$ & 51 & $\mathrm{Cl}(\mathrm{L})$ & 318 & Broca \\
PWA19 & $\mathrm{m}$ & 57 & $\mathrm{Cl}(\mathrm{L})$ & 122 & Global \\
PWA20 & $\mathrm{f}$ & 39 & $\mathrm{Cl}(\mathrm{L})$ & 160 & Anomic \\
PWA21 & $\mathrm{m}$ & 68 & $\mathrm{Cl}(\mathrm{L})$ & 14 & Global \\
PWA22 & $\mathrm{m}$ & 59 & $\mathrm{Cl}(\mathrm{L})$ & 71 & Broca \\
PWA25 & $\mathrm{m}$ & 67 & $\mathrm{Cl}(\mathrm{L})$ & 267 & Broca \\
PWA26 & $\mathrm{m}$ & 46 & $\mathrm{Cl}(\mathrm{L})$ & 57 & Wernicke \\
PWA27 & $\mathrm{m}$ & 54 & $\mathrm{Cl}(\mathrm{L})$ & 156 & Broca \\
PWA28 & $\mathrm{f}$ & 48 & $\mathrm{Cl}(\mathrm{THA})$ & 245 & not class. \\
PWA31 & $\mathrm{f}$ & 59 & $\mathrm{Cl}(\mathrm{L})$ & 16 & Global \\
PWA32 & $\mathrm{m}$ & 66 & $\mathrm{Cl}(\mathrm{L})$ & 20 & Broca \\
PWA33 & $\mathrm{f}$ & 51 & $\mathrm{Cl}(\mathrm{L})$ & 40 & not class. \\
PWA34 & $\mathrm{f}$ & 52 & $\mathrm{Cl}(\mathrm{L})$ & 39 & Conduction \\
PWA35 & $\mathrm{m}$ & 36 & $\mathrm{Cl}(\mathrm{L})$ & 40 & Global \\
PWA37 & $\mathrm{f}$ & 55 & $\mathrm{Cl}(\mathrm{L})$ & 12 & Wernicke \\
\hline
\end{tabular}

Note. $\mathrm{Cl}=$ cerebrovascular infarction. $\mathrm{TBI}=$ traumatic brain injury. $\mathrm{L}=$ left hemisphere. $\mathrm{R}=$ right hemisphere THA = thalamus. Not class. $=$ not classifiable .

Several tests were administered to examine the PWA's language capacities and their aphasia severities (Aachen Aphasia Test, AAT, Huber, Poeck, Weniger, \& Willmes, 1983), their nonverbal semantic processing skills (Bogenhausener Semantics Examination, BOSU, Glindemann, Klintwort, Ziegler, \& Goldenberg, 2002) and the potential presence of limb apraxia (Test of Upper Limb Apraxia, TULIA, Vanbellingen et al., 2010). The PWA presented with different types of aphasia (see Table 1). Seven PWA showed limitations of their verbal processing abilities and three participants' test results indicated limb apraxia. More detailed information on the PWA can be found in De Beer et al. (2019).

The study received ethical approval by the Ethics Committee of the medical council Westfalen-Lippe and the Faculty of Medicine, University of Münster, Germany (AZ 2013356-f-S).

\subsection{Experimental procedure}

Data on the production of gesture and speech were assessed in two different conditions, each with different communicative constraints. The first condition was the spontaneous conversation about four topics of daily living. The investigator (the first author) addressed all four topics by asking the participants very general as well as more specific 
questions. Within the four topics, participants were free to decide what they would talk about in the conversation. The investigator supported the participants by ensuring comprehension and by supporting the PWA in cases of disfluencies, if possible. The duration of the whole conversation was set to approximately 20 minutes.

The second condition was the narration task, in which the participants were asked to retell five short fragments from the Sylvester and Tweetybird cartoon Canary Row (Warner Brothers Entertainment). These cartoon clips have been frequently used to study the production of gestures, since their clear structure and salient animations tend to facilitate the production of gestures (McNeill, 1992). Furthermore, the content of the cartoons is revealed by visual and sound information and not by spoken utterances, making the cartoons suitable stimuli for the PWA. The participants watched the five cartoon clips on a laptop computer with a 15.6-inch screen. They were instructed to retell the cartoons as informatively as possible and assume that the listener was unfamiliar with the cartoons' content. The investigator did not support the participants with their narrations and informed them accordingly before the task. The first of the five cartoons served as a practice trial to ensure the participants' full understanding of the task.

Both communicative tasks were recorded with two Panasonic HD-cameras, one capturing both interlocutors and one only recording the participant. The participants were always asked to address the investigator to distract their primary focus of attention from the cameras.

\subsection{Data Analysis}

We analysed a total of ten minutes of video data from the spontaneous conversations. Two five-minute-fragments were extracted as follows: the first extract always started 2 minutes after the beginning of the conversation, when participants spoke about their work and heritage. The second extract was taken from the second half of the conversation, when participants spoke about leisure and holidays. We always excluded the first two minutes of the spontaneous conversations from the analyses, since participants were expected to need some time to familiarize themselves with the experimental situation. We analysed two cartoon narrations (of cartoons 3 and 4) from each participant.

Ten video clips (four clips from spontaneous conversations and six cartoons narrations) had to be excluded from the analysis due to insufficient visibility of the participants' arms and hands or acoustic disturbances. Additionally, the narrations of two PWA could not be analysed since they refused to or were unable to complete the task due to serious problems in memorizing and recalling the content of the cartoons.

We used the annotation software ELAN (Wittenburg, Brugman, Russel, Klassmann, \& Sloetjes, 2006) to annotate and transcribe speech and gestures produced by the participants. To assess spoken production, we counted all words produced by the participants, excluding interjections, filler words, word fragments and elements of automatic speech produced by the PWA.

We annotated the gestures produced by the participants without sound and in slow motion ( $50 \%$ of regular speed). Firstly, gestures were identified, i.e. distinguished from movements of the arms and hands that did not carry any representational meaning (i.e. selfmanipulations). Secondly, gestures were annotated and classified into different categories according to their main characteristics (see De Beer et al. (2019) for descriptions and 
analyses of the different gesture types). In this study we only looked at iconics (see definition above).

We transformed the absolute numbers of iconics produced into the relative measure of gestures per 100 words (gesture-to-word-ratio). We did not include the gesture-to-wordratios for two of the PWA (PWA07 and PWA19), because they produced hardly any identifiable words, which would disproportionally warp the gesture per 100 words measure. These two participants were removed from subsequent statistical analyses ${ }^{2}$.

\subsection{Interrater reliability}

Gestures were identified and classified by the first author of the paper. A second rater independently classified gestures in $16 \%$ of the video data (controlled for participant groups and conditions) to examine interrater reliability. We found strong agreement between the two raters (Cohen's Kappa $=0.8$ ).

\section{Results}

We employed a conventional repeated measures ANOVA with group as a betweensubjects factor and task as a within-subjects factor and with iconics/100 words as the dependent variable. We also conducted a Bayesian repeated measures ANOVA because this kind of analysis also allows quantification of the relative evidence for the null hypothesis over the alternative hypothesis. Furthermore, paired t-tests (conventional and Bayesian) were used to analyse within-subject effects with iconics/100 words as the dependent variable.

Table 2. Descriptives for iconics/100 words for Participants with Aphasia (PWA) and Control Participants (CP), and for both Conversation and Narration tasks. Mean iconic gestures per 100 words (standard deviation).

\begin{tabular}{lcc} 
& Conversation & Narration \\
\cline { 2 - 3 } PWA & $9.53(9.57)$ & $29.22(18.66)$ \\
CP & $2.93(2.30)$ & $5.96(4.03)$ \\
\hline
\end{tabular}

The means and standard deviations of the iconic gestures per 100 words for the two groups and the two tasks are summarized in Table 2 and the iconic gestures per 100 words of each individual PWA in both tasks are visualized in Fig. 3.

\footnotetext{
${ }^{2}$ Additional analyses revealed that their removal did not change the pattern of the reported results.
} 


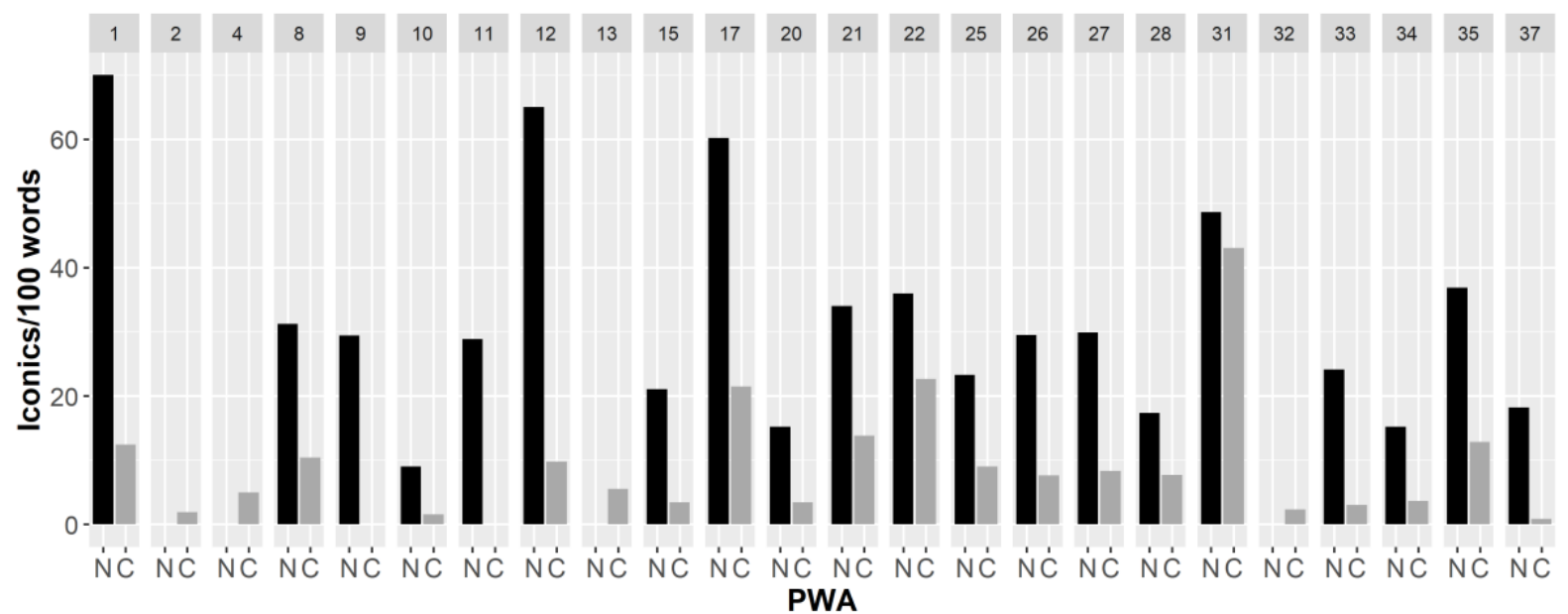

Figure 3. Iconics per 100 words produced by each individual PWA in the narration task $(\mathrm{N}$, black bars) and in conversation ( $\mathrm{C}$, grey bars). Missing data points: Data from PWA04 and PWA13 is missing for the narration due to abandonment of the task and data from PWA09 and PWA11 are missing for conversation due to blocked visibility of hand movements in the videos.

A conventional repeated measures ANOVA revealed main effects of group $(F(1,43)=$ $30.197, p<.001)$ and task $(F(1,43)=47.170, p<.001)$ on the rates of iconics per 100 words. We further found an interaction of the factors group and task $(F(1,43)=25.592, p<.001)$. See Fig. 4 for an illustration of the interaction.

The Bayesian analysis (Bayesian repeated measures ANOVA) revealed extreme evidence for an effect of Task $\left(\mathrm{BF}_{10}=2501.348\right)$ as well as for an effect of Group $\left(\mathrm{BF}_{10}=\right.$ 3079.808). The model including a Group/Task interaction is the most predictive model $\left(\mathrm{BF}_{10}\right.$ $=2.318 \mathrm{e}+10)$ and is more predictive than the model without the interaction $\left(\mathrm{BF}_{10}=1.572 \mathrm{e}\right.$ +7) (see Table 3).

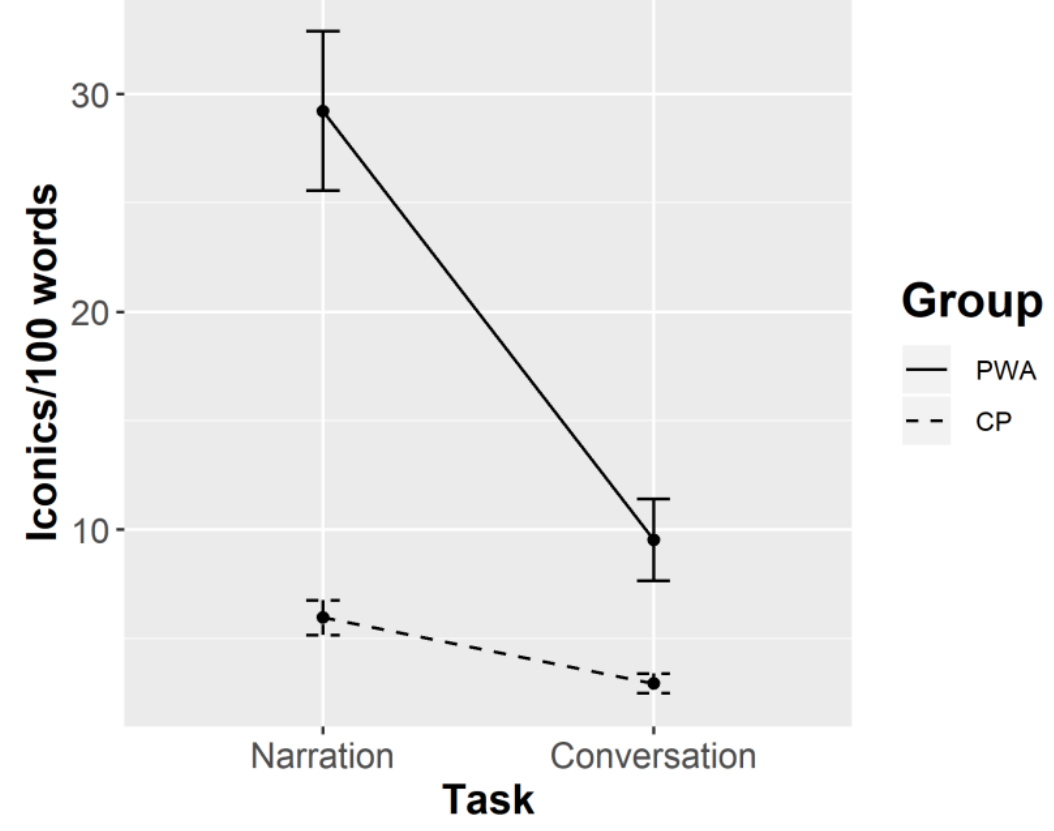


Figure 4. Iconics per 100 words produced by both participant groups in two conditions with main effects of group and task. PWA = Participants with aphasia. $\mathrm{CP}=$ Control Participants.

Table 3. Model comparisons of Bayesian analyses for iconics/100 words.

\begin{tabular}{lrcrrr}
\hline \multicolumn{1}{c}{ Models } & $\mathbf{P ( M )}$ & $\mathbf{P}(\mathbf{M} \mid$ data) & $\mathbf{B F}_{\mathbf{M}}$ & $\mathbf{B F}_{\mathbf{1 0}}$ & error \% \\
\hline Null model (incl. subject) & 0.200 & $4.311 \mathrm{e}-11$ & $1.724 \mathrm{e}-10$ & 1.000 & \\
Task + Group + Task * Group & 0.200 & 0.999 & 5896.013 & $2.318 \mathrm{e}+10$ & 1.987 \\
Task + Group & 0.200 & $6.777 \mathrm{e}-4$ & 0.003 & $1.572 \mathrm{e}+7$ & 1.736 \\
Task & 0.200 & $1.078 \mathrm{e}-7$ & $4.313 \mathrm{e}-7$ & 2501.348 & 2.450 \\
Group & 0.200 & $1.328 \mathrm{e}-7$ & $5.311 \mathrm{e}-7$ & 3079.808 & 0.884 \\
\hline
\end{tabular}
Factor.)

Note. All models include the factor subject. $(\mathrm{P}=$ Probability. $\mathrm{M}=$ Model. $\mathrm{BF}=$ Bayes

We also looked at the within-subject effect of task for both groups separately. In the PWA, a conventional paired t-test revealed a significant effect of task $(t(19)=5.481, p=$ $<.001)$. The Bayesian paired t-test revealed extreme evidence with a Bayes factor $\left(\mathrm{BF}_{10}\right)$ of 1062.237. For the $C P$, the paired samples t-test revealed a significant task effect $(t(24)=$ 4.193, $p=<.001$ ), which is supported by the strong evidence found in the Bayesian paired $t$ test $\left(\mathrm{BF}_{10}=94.130\right)$.

\section{Discussion}

In both groups, the narration task evoked a higher gesture-to-word ratio than the spontaneous conversation. This is not too surprising, as the cartoon narration task contains a lot of dynamic imagery, which is the reason it is the most commonly used task to evoke spontaneous iconic gestures (McNeill, 1992; McNeill \& Levy, 1982). However, the difference was much larger in the PWA group: we found a significant interaction of task and group for the gesture-to-word-ratios of iconic gestures, such that the increase of gestures/100 words for the narrations in the PWA was much larger than for the $\mathrm{CP}^{3}$.

Our current results clearly suggest that PWA employed iconics to compensate for their verbal limitations and, as predicted, this effect was larger in the narration task, because in that task they had to express pre-determined and sometimes hard-to-verbalize content. The compensatory use of iconics is moderated by the communicative constraints of the task, i.e. higher constraints lead to a more frequent use of iconics by the PWA. Our findings are therefore not in line with the contention that gesture and speech are impaired to the same extent in persons with aphasia (Duffy \& Duffy, 1981; McNeill, 1992). Instead, they are in agreement with studies supporting the notion of compensatory gesture use in

${ }^{3}$ These statistics are different from those in De Beer et al. (2019) for two reasons. First, in that study, the dependent variable was the gesture rate per minute instead of per 100 words, which lead to partly different results, because the speech rate differs between the two participant groups. Therefore, the rates of iconics per 100 words also differ from the rates of iconic gestures per minute. Second, De Beer et al. (2019) analyzed the iconic gestures and the pantomime gestures separately, whereas in this study they are combined into one group. 
PWA (De Beer et al., 2017; Herrmann et al., 1988; Hogrefe et al., 2013; Hogrefe, Ziegler, Weidinger, \& Goldenberg, 2012; van Nispen, van de Sandt-Koenderman, Sekine, Krahmer, \& Rose, 2017; Wilkinson et al., 2010).

\subsection{Evaluation of models}

\subsubsection{Original Sketch Model (De Ruiter, 2000)}

The results of the current analyses for the PWA are compatible with the original Sketch Model by De Ruiter (2000). Following De Ruiter and De Beer (2013), we assume that in PWA the conceptualizer adapts to the underlying deficit in the speech channel. PWA therefore produce more iconics than people without language difficulties, expressing more information in the gesture channel, resulting in a higher gesture-to-word ratio. The grouptask-interaction we found in the current study clearly indicates that PWAs' compensatory use of iconics is not only higher than for CP in general, but is also proportionally much higher in the narration task, which is the task with higher communicative constraints. In addition to the explanation suggested by De Ruiter and De Beer (2013), our current results lead to the following conclusions. First, this explanation is not only true for people with nonfluent aphasias, as discussed by De Ruiter and De Beer, but holds more generally, i.e. for a group of PWA with varying types and severities of aphasias. Second, the compensatory use of gestures by PWA is more evident in a task with higher communicative constraints, in which the complexity of the content to be expressed was predefined as opposed to spontaneous conversation. So, it appears that the conceptualizer reacts to increasing difficulties in the spoken modality by generating relatively more iconic gestures, as the Sketch model predicts.

One central question regarding the production of gesture and speech by PWA is, whether the gestural compensation by PWA is a conscious strategy. Levelt (1989) assumes that conscious control of the speech production process within his model is only possible at the stage of the conceptualizer, without implying that the conceptualisation process is under complete conscious control. Certain aspects of this process are partially automated, and the underlying planning and production processes are not subject to conscious control. Furthermore, feedback and repair processes can evoke conscious control. In line with this, we assume that in PWA, the regular processes of planning and producing gestures and speech are not consciously controlled, since they are subject to a general adaptation to the deficit (De Ruiter \& De Beer, 2013). However, in cases of more severe problems in speech production, the conceptualizer is informed about the current problems via the two feedback loops that the Sketch model inherited from Levelt's 1989 model. The subsequent process of reformulation will be subject to more conscious control, which can in turn lead to the decision to shift some of the expressive load to the gesture modality and e.g. plan an iconic gesture to refer to an object or concept in the case of a word finding difficulty.

Importantly, our assumptions on the relationship of gesture and speech in PWA only hold for PWA without severe impairments in executive functioning, including executive control and cognitive flexibility. Limitations in cognitive flexibility and executive functions limit the ability of a flexible use of different communicative modalities (Purdy, 2002; Spitzer, Binkofski, Willmes, \& Bruehl, 2019). Therefore, we would assume PWA with such deficits to struggle with the effective change of modalities, i.e. to compensate for their verbal impairments by an increased use of gestures. 
In our clinical assessment (see Table 1 in de Beer et al., 2019), participant PWA02, showed impaired semantic processing capacities. This might have prevented the retrieval of relevant information from memory. Furthermore, two of the participants (PWA02, PWA32) were diagnosed with having limb apraxia (see Table 1 in de Beer et al., 2019) which probably limited their abilities to produce identifiable iconic gestures (see e.g., Hogrefe et al., 2012). Indeed, in the two mentioned PWA the use of iconic gestures was very low in conversation as well as in the narration task (see Fig. 3). We therefore assume a general deficit in using the gestural modality effectively in these two participants.

For the control participants, the rate of iconics per word was much lower than for the PWA in both tasks, and more importantly, the increase of the rate of iconics per word in the narration task as compared to spontaneous conversation was much smaller in the CP as compared to the PWA. For the participants of the control group, the conceptualizer is not faced with severe limitations in the speech channel and a transfer of information to the gesture channel is therefore not required. While the data from the CP does not provide direct evidence in favor of the AR-Sketch model, as pointed out above, the assumption of the original Sketch Model that gesture compensates for expressive difficulties in speech production in people without language impairments has been falsified by a range of central empirical findings on the relation of iconic gestures with their accompanying speech (De Ruiter et al., 2012; De Ruiter, 2017; Kita \& Özyürek, 2003; So et al., 2009).

\subsubsection{AR-Sketch Model (De Ruiter, 2017)}

In contrast to the original Sketch Model, the AR-Sketch Model assumes no adaptive or tradeoff relationship between the speech and the gesture channel, but an asymmetric redundancy between the two modalities with speech being the dominant channel. This core assumption of the AR-Sketch Model is very much in line with the production of iconics found for the control participants of the current study. The relation between iconic gesture and speech was found to be largely independent of the task and the respective communicative constraints in persons without language impairments. The small significant effect of task within the control group could have been caused by the high number of spatial/dynamic events in the cartoon stimuli. That is, as in De Ruiter, Bangerter \& Dings (2012) there was little evidence that the control participants made extensive compensatory use of iconics in either communicative task.

Explaining our findings with the PWA using the AR-Sketch Model is much harder than with the original Sketch Model. The AR-Sketch Model does not allow for a transfer of information from one modality to the other, because iconic gestures are assumed to only express information that is redundant to the accompanying speech. Furthermore, speech is assumed to be the dominant modality and the production of iconic gestures is planned based on only a subset of the information that is available for speech production. According to the AR-Sketch Model PWA have no opportunity to express non-redundant and speechreplacing information by the use of iconic gestures. However, there is evidence suggesting that PWA are able to employ gestures, including iconics for the expression of content that complements and is even essential for the intelligibility of the spoken utterance (De Beer et al., 2017; De Beer et al., 2018; Dipper et al., 2015; van Nispen et al., 2017). 


\subsubsection{Alternative models of gesture production}

There are two other often-cited models of gesture and speech production that we need to briefly discuss here. First, the "Lexical Access model" by Krauss, Chen \& Gottesmann (2000), assumes that gestures are produced by speakers directly from imagistic representations in short-term-memory in order to activate or "cross-modally prime" relevant representations that would facilitate the process of word-form retrieval. While this at first sight appears to be relevant in the context of aphasia, there are two reasons why this model is not adequate to explain our results. First, it assumes that gestures correspond to individual words, whereas there is abundant evidence that the information represented in iconic gestures is equivalent to higher-level semantic (Clark, 1996; De Ruiter, 2000; Kendon, 1994; Kendon, 2004) or syntactic (Clark, 1996; Kita \& Özyürek, 2003) representations. More importantly, however, there are two studies (Beattie \& Coughlan, 1999; Holler et al., 2013) showing that making gestures does not help speakers to access hard-to-find words (for a more extensive discussion, see De Ruiter, 2006 and De Ruiter \& De Beer, 2013).

Another model that is relevant in this context is the "Interface Model" by Kita \& Özyürek (2003). This model is very similar to the Sketch model, and inherits the latter's assumption that the conceptualizer decides which modalities are going to be used for expressing the content in the preverbal message (ibid, p. 28). Also, even though the diagram depicting the model looks different from that of the Sketch model, it contains essentially the same conceptualizer, which is represented by three boxes (Communication Planner, Message Generator, and Action Generator) instead of the one box (Conceptualiser) in the Sketch model (see De Ruiter \& De Beer, 2013 for a more detailed discussion). The difference between the Sketch Model and the Interface Model is in how they determine the shape of iconic gestures. In the Sketch Model, this is directly based on imagery in short term memory, whereas in the Interface Model, it is the result of an iterative negotiation process between the expressive capabilities of the action generator (gesture), the message generator (speech) and the communicative intention (Kita \& Özyürek, 2003, pp. 28-29). Hence, for the purposes of this paper, the Interface model would make the same predictions as the Sketch model.

\subsubsection{Summary}

To summarize, our results show that the production of iconic gestures by PWA is in line with the predictions of the original Sketch Model (or with the functionally equivalent Interface model), and not with the AR-Sketch model. Evidence from a number of earlier studies on neurotypical speakers, has suggested that the use of iconics by people without language impairments is more adequately modelled by the AR-Sketch Model (De Ruiter, 2017). These outcomes of our model comparison demonstrate that theories about the relationship of gesture and speech in people without language impairments are not necessarily applicable for PWA. The relation between the speech and gesture modality in PWA seems to differ from people without language impairments, hence neither of the two models can explain the gesture production of both participant groups. Future modeling work could be aimed at creating a unified model that incorporates both people with aphasia as well as neurotypical communicators. One avenue that could be explored for such a unified model is to modify the conceptualizer in the AR-Sketch model in such a way that when it detects problems with speech (through the internal and/or external self-monitoring 
loop; see Levelt, 1989 for details), it switches to "gesture only" production of the currently active communicative intention. Such an extension of the model would also accommodate other common communicative challenges, like speaking in highly noisy environments, or communicating in a non-native language.

\subsection{Limitations}

We acknowledge that we included participants in our group of PWA with etiologies other than stroke, including one participant with hypoxia (PWA02) and one participant with TBI (PWA04). We acknowledge that these participants should appropriately be described as having cognitive communication disorders rather than aphasia (e.g., MacDonald \& Johnson, 2005). PWA04 also displayed cognitive and visual processing deficits which kept her from completing the narration task. PWA02 was found to have a semantic processing deficit as well as limb apraxia and had a general deficit in the use of iconic gestures as described above. The evaluation of cognitive impairments and their influence on multimodal communication in PWA was not within the scope of the current study. Since literature focusing on the influence of cognitive disorders on communicative processes suggest that e.g. executive functions impact on multimodal communication (Hogrefe \& De Beer, 2018; Purdy, 2002; Spitzer et al., 2019), future studies should take these aspects into account more carefully. This holds not only for the two participants mentioned above, but for PWA in general, since they often present with additional cognitive impairments (Hogrefe et al., 2012; Spitzer et al., 2019).

Furthermore, we did not control for a potential influence of multimodal communication therapy on the PWA's performance in the current study. We acknowledge that a systematic training in multimodal communication or more specifically in the use of communicative gestures potentially impacts the spontaneous gesture production in PWA. However, since our task effects were analysed within and not between cases, we do not assume an effect of potential communicative therapy in some of the tested PWA on our reported results.

The two tasks employed in the current study differed with respect to a range of aspects, which we are not able to tear apart with respect to our results. One central aspect is certainly the visual information of the cartoons shown in the narration task, which is known to elicit the use of iconics (Hostetter \& Skirving, 2011; McNeill, 1992) and was not present in the spontaneous conversation. However, since both tasks were used in both participant groups, the results from these tasks are still informative when comparing the two groups.

We acknowledge that the two models of gesture production discussed in the current study were not designed to model the production of gesture and speech in PWA, but only in people without language impairment. It is still a noteworthy and interesting finding that one of the models better reflects the use of iconics in PWA than the other model. To completely account for the complex relationship of gesture and speech in PWA, more specific theories and models are needed to fully capture PWA's multimodal communication behaviour. Modeling gesture and speech in PWA would be greatly facilitated by future research that would include in-depth qualitative analyses of the iconics used by PWA and CP in the two communicative tasks and their relationship with the accompanying speech. However, the 
core assumptions of the Sketch Model (De Ruiter, 2000) seem to be supported by the use of gesture in the majority of PWA in the current study.

\section{Conclusion}

We showed in an experiment that compared the gesture-to-word ratio in persons with aphasia (PWA) of varying degrees of severities and control persons without language impairments that the compensatory use of iconic gestures is largest for the PWA in the narration task where they had to express pre-determined content and did not have the possibility to choose topics. Earlier work has shown that speakers without language impairment do not show this compensation effect. It was the latter finding that has motivated the small but fundamental change in the original Sketch model, resulting in the new AR-Sketch model. So, the old model seems to predict the gesture behavior of the PWA, whereas the new model does not. It appears that the original Sketch model, although it was falsified by a number of important results from studies with neurotypical participants, is still the most adequate model for the study of impaired speech production (see also De Ruiter \& De Beer, 2013). These conclusions hold for most of our studied PWA, excluding PWA with severe cognitive deficits or with limb apraxia.

Our findings suggest that while communicating with others, persons with aphasia and people without language impairment use iconic gestures in fundamentally different ways. While people without language impairment use gesture as a largely redundant signal enhancer (De Ruiter, Bangerter \& Dings, 2012), people with aphasia use it as an alternative communicative channel to get their communicative intention across (De Beer et al., 2017; Hogrefe et al., 2013; Hogrefe et al., 2017; Rose et al., 2017). One reason that this is intriguing is that speech production is generally assumed to be a largely automatic process (Levelt, 1989) and iconic gesture to be coupled with speech at a deep level (McNeill, 1985; McNeill, 1992). Our results suggest that even at a later age, i.e. after the first language has been acquired in full, the functional relation between gesture and speech can be changed to support additional communicative mechanisms. In other words, the speaking and gesturing processes are more under our control than is often assumed (see also Goodwin 2000). This, in turn, suggests that both speaking and gesturing are sub-processes of a separate, higherlevel cognitive process concerned with general communication, a process that in our view deserves more attention in the Cognitive Sciences.

\section{Acknowledgements}

Carola de Beer was funded by a PhD scholarship of the University of Bielefeld; Katharina Hogrefe was funded by the Federal Ministry of Education and Research (BMBF; grant 01UL1822x). 


\section{References}

Alibali, M. W., Heath, D. C., \& Myers, H. J. (2001). Effects of Visibility between Speaker and Listener on Gesture Production: Some Gestures Are Meant to Be Seen. Journal of Memory and Language, 44(2), 169-188.

Alibali, M. W., Kita, S., \& Young, A. J. (2000). Gesture and the process of speech production: We think, therefore we gesture. Language and Cognitive Processes, 15(6), 593-613.

Anglade, C., Le Dorze, G., \& Croteau, C. (2020). How clerks understand the requests of people living with aphasia in service encounters. Clinical linguistics \& phonetics, 1-16.

Beattie, G., \& Coughlan, J. (1999). An experimental investigation of the role of iconic gestures in lexical access using the tip-of-the-tongue phenomenon. British Journal of Psychology, 90, 35-56.

Beattie, G., \& Shovelton, H. (2011). An exploration of the other side of semantic communication: How the spontaneous movements of the human hand add crucial meaning to narrative. Semiotica, 184, 33-51.

Butterworth, B., \& Hadar, U. (1989). Gesture, speech, and computational stages: a reply to McNeill. Psychological Review, 96, 168-174.

Cicone, M., Wapner, W., Foldi, N., Zurif, E., \& Gardner, H. (1979). The relation between gesture and language in aphasic communication. Brain and Language, 8, 324-359.

Clark, H. H. (1996). Using Language (1. Auflage). Cambridge: Cambridge University Press.

Damico, J. S., Wilson, B. T., Simmons-Mackie, N. N., \& Tetnowski, J. A. (2008). Overcoming unintelligibility in aphasia: the impact of non-verbal interactive strategies. Clinical linguistics \& phonetics, 22(10-11), 775-782.

De Beer, C., Carragher, M., van Nispen, K., Hogrefe, K., De Ruiter, J. P., \& Rose, M. L. (2017). How much information do people with aphasia convey via gesture? American Journal of Speech Language Pathology, 26(2), 483-497.

De Beer, C., De Ruiter, J. P., Hielscher-Fastabend, M., \& Hogrefe, K. (2019). The production of gesture and speech by people with aphasia: Influence of communicative constraints. Journal of Speech, Language and Hearing Research.

De Beer, C., Hogrefe, K., \& De Ruiter, J. P. (2018). Features of semantic content expressed via gesture by people with aphasia. Aphasiology, 32(1), 18-20.

De Ruiter, J. P. (2000). The production of gesture and speech. In D. McNeill (Ed.), Language and Gesture (pp. 284-311). Cambridge: Cambridge University Press.

De Ruiter, J. P. (2017). The asymmetric redundancy of gesture and speech. In R. B. Chruch, M. W. Alibali, \& S. D. Kelly (Eds.), Why gesture? How the hands function in speaking, thinking and communicating (59-75). Amsterdam: John Benjamins Publishing Company.

De Ruiter, J. P. (2006). Can gesticulation help aphasic people speak, or rather, communicate? Advances in Speech-Language-Pathology, 8, 124-127.

De Ruiter, J. P., Bangerter, A., \& Dings, P. (2012). The Interplay Between Gesture and Speech in the Production of Referring Expressions: Investigating the Tradeoff Hypothesis. Topics in Cognitive Science, 4(2), 232-248. 
De Ruiter, J. P., \& De Beer, C. (2013). A critical evaluation of models of gesture and speech production for understanding gesture in aphasia. Aphasiology, 27(9), 1015-1030.

Dipper, L., Pritchard, M., Morgan, G., \& Cocks, N. (2015). The language-gesture connection: Evidence from aphasia. Clinical linguistics \& phonetics, 29(8-10), 748-763.

Duffy, R. J., \& Liles, B. Z. (1979). A translation of Finkelnburg's (1870) lecture on aphasia as "asymbolia" with commentary. The Journal of speech and hearing disorders, 44(2), 156168.

Duffy, R. J., \& Duffy, J. R. (1981). Three Studies of Deficits in Pantomimic Expression and Pantomimic Recognition in Aphasia. Journal of Speech, Language, and Hearing Research, 24(1), 70-84.

Feyereisen, P. (1983). Manual Activity During Speaking in Aphasic Subjects. International Journal of Psychology, 18(1-4), 545-556.

Glindemann, R., Klintwort, D., Ziegler, W., \& Goldenberg, G. (2002). Bogenhausener Semantik-Untersuchung: BOSU (1. Auflage). München: Urban und Fischer.

Goldin-Meadow, S. (1999). The role of gesture in communication and thinking. Trends in Cognitive Sciences, 3(11), 419-429.

Herrmann, M., Reichele, T., Lucius-Hoene, G., Wallesch, C.-W., \& Johannsen-Horbach, H. (1988). Nonverbal communication as a compensative strategy for several nonfluent aphasics? - A quantitative approach. Brain and Language, 33, 41-54.

Hogrefe, K., \& De Beer, C. (2018). Aktuelle Ansätze zur Diagnostik und Therapie kommunikativer Fähigkeiten bei Personen mit Aphasie. Neurologie \& Rehabilitation, 24(2), 130-142.

Hogrefe, K., Ziegler, W., Weidinger, N., \& Goldenberg, G. (2012). Non-verbal communication in severe aphasia: Influence of aphasia, apraxia, or semantic processing? Cortex, 48(8), 952-962.

Hogrefe, K., Ziegler, W., Weidinger, N., \& Goldenberg, G. (2017). Comprehensibility and neural substrate of communicative gestures in severe aphasia. Brain and Language, 171, $62-71$.

Hogrefe, K., Ziegler, W., Wiesmayer, S., Weidinger, N., \& Goldenberg, G. (2013). The actual and potential use of gestures for communication in aphasia. Aphasiology, 27(9), 10701089.

Holler, J., Turner, K., \& Varcianna, T. (2013). It's on the tip of my fingers: Co-speech gestures during lexical retrieval in different social contexts. Language and Cognitive Processes, 28(10), 1509-1518.

Hostetter, A. B., \& Skirving, C. J. (2011). The Effect of Visual vs. Verbal Stimuli on Gesture Production. Journal of Nonverbal Behavior, 35(3), 205-223.

Huber, W., Poeck, K., Weniger, D., \& Willmes, K. (1983). Aachener Aphasie Test (AAT). Göttingen: Hogrefe.

Kendon, A. (1994). Do Gestures Communicate?: A review. Research on Language \& Social Interaction, 27(3), 175-200.

Kendon, A. (2004). Gesture: Visible action as utterance. Cambridge: Cambridge University Press. 
Kita, S., \& Özyürek, A. (2003). What does cross-linguistic variation in semantic coordination of speech and gesture reveal?: Evidence for an interface representation of spatial thinking and speaking. Journal of Memory and Language, 48, 16-32.

Kong, A. P.-H., Law, S.-P., Wat, W. K.-C., \& Lai, C. (2015). Co-verbal gestures among speakers with aphasia: Influence of aphasia severity, linguistic and semantic skills, and hemiplegia on gesture employment in oral discourse. Journal of Communication Disorders, 56, 88102.

Krauss, R. M., Chen, Y., \& Gottesman, R. F. (2000). Lexical Gestures and Lexical Access: A Process Model. In D. McNeill (Ed.), Language and Gesture (pp. 261-283). Cambridge: Cambridge University Press.

Krauss, R. M., Dushay, R. M., Chen, Y., \& Rauscher, F. (1995). The communicative value of conversational hand gestures. Journal of Experimental Social Psychology, 31, 533-552.

Krauss, R. M., Morrel-Samuels, P., \& Colasante, C. (1991). Do conversational hand gestures communicate? Journal of Personality and Social Psychology, 61(5), 743-754.

Levelt, W. J. M. (1989). Speaking. Cambridge [u.a.]: MIT Press.

Lott, P. (1999). Gesture and Aphasia. Bern: Peter Lang.

MacDonald, S., \& Johnson, C. J. (2005). Assessment of subtle cognitive-communication deficits following acquired brain injury: A normative study of the Functional Assessment of Verbal Reasoning and Executive Strategies (FAVRES). Brain injury, 19(11), 895-902.

McNeill, D. (1985). So you think gestures are nonverbal? Psychological Review, 92(3), 350371.

McNeill, D. (1992). Hand and mind: What gestures reveal about thought. Chicago: University of Chicago Press.

McNeill, D., \& Duncan, S. D. (2000). Growth points and thinking-for-speaking. In D. McNeill (Ed.), Language and Gesture (pp. 141-161). Cambridge: Cambridge University Press.

McNeill, D., \& Levy, E. T. (1982). Conceptual representations in language activity and gesture. In R. Jarvella \& W. Klein (Eds.), Speech, place, and action. Chichester: Wiley \& Sons.

Purdy, M. (2002). Executive function ability in persons with aphasia. Aphasiology, 16(4-6), 549-557.

Rose, M. L., Mok, Z., \& Sekine, K. (2017). Communicative effectiveness of pantomime gesture in people with aphasia. International Journal of Language \& Communication Disorders, 52, 227-237.

Sekine, K., \& Rose, M. L. (2013). The Relationship of Aphasia Type and Gesture Production in People With Aphasia. American Journal of Speech-Language Pathology, 22(4), 662-672.

Sekine, K., Rose, M. L., Foster, A. M., Attard, M. C., \& Lanyon, L. E. (2013). Gesture production patterns in aphasic discourse: In-depth description and preliminary predictions. Aphasiology, 27(9), 1031-1049.

So, W. C., Kita, S., \& Goldin-Meadow, S. (2009). Using the hands to identify who does what to whom: Gesture and speech go hand-in-hand. Cognitive Science, 33, 115-125.

Spitzer, L., Binkofski, F., Willmes, K., \& Bruehl, S. (2019). Executive functions in aphasia: A novel aphasia screening for cognitive flexibility in everyday communication.

Neuropsychological rehabilitation, 1-19. 
van Nispen, K., van de Sandt-Koenderman, W. M. E., Sekine, K., Krahmer, E., \& Rose, M. L. (2017). Part of the message comes in gesture: How information is conveyed in gesture as compared to speech by people with aphasia. Aphasiology, 31(9), 1078-1103.

Vanbellingen, T., Kersten, B., van Hemelrijk, B., van de Winckel, A., Bertschi, M., Müri, R., et al. (2010). Comprehensive assessment of gesture production: a new test of upper limb apraxia (TULIA). European Journal of Neurology, 17(1), 59-66.

Wilkinson, R., Beeke, S., \& Maxim, J. (2010). Formulating Actions and Events With Limited Linguistic Resources: Enactment and Iconicity in Agrammatic Aphasic Talk. Research on Language \& Social Interaction, 43(1), 57-84.

Wittenburg, P., Brugman, H., Russel, A., Klassmann, A., \& Sloetjes, H. (2006). ELAN: a Professional Framework for Multimodality Research. Proceedings of LREC 2006, Fifth International Conference on Language Resources and Evaluation, Genoa, Italy, from http://www.Irec-conf.org/proceedings/Irec2006/. 